\title{
Hemangioma cavernoso difuso do útero
}

\author{
Diffuse cavernous hemangioma of the uterus
}

Alessandra Guerra Godoy ${ }^{1}$

Giovana Boscato²

\begin{tabular}{l|l}
\multicolumn{1}{c|}{ Unitermos } & res umo \\
Hemangioma & $\begin{array}{l}\text { O hemangioma cavernoso difuso do útero é uma condição rara, muitas vezes subdiagnosticado, por não } \\
\text { apresentar um quadro clínico típico. É uma lesão hamartomatosa, onde a parede miometrial é total ou } \\
\text { Cavernoso }\end{array}$ \\
$\begin{array}{l}\text { parcialmente substituída por uma proliferação de vasos arteriais e venosos, com formação fistular entre si. } \\
\text { Os autores relatam o caso de uma paciente em que o diagnóstico de hemangioma cavernoso difuso } \\
\text { uterino foi um achado ocasional, uma vez que a paciente não apresentava sintomas clínicos. }\end{array}$ \\
$\begin{array}{l}\text { Útero } \\
\text { Anatomopatológico }\end{array}$
\end{tabular}

abstract

The diffuse cavernous hemangioma of the uterus is a rare condition, and many times underdiagnosed by not showing a typical clinical picture. It is an hamartomatous lesion, where the myometrial wall is totally or partially replaced by a proliferation of arteries and veins, occurring fistulas among themselves. The authors present the case of a patient whose diagnosis of diffuse cavernous hemangioma of the uterus was made through an occasional finding, once the patient had no clinical symptoms.

key words

Hemangioma

Cavernous

Diffuse

Uterus

Anatomopathologic

\section{Introdução}

Tumores vasculares dificilmente ocorrem no trato genital feminino, sendo predominantes dentro do corpo uterino (1). O hemangioma cavernoso difuso uterino constitui uma condição rara na qual a parede miometrial é invadida por uma lesão hamartomatosa, composta por vasos peculiares, dilatados, com delicado envoltório endotelial, derivado do tecido conectivo (2). É uma lesão circunscrita, grande, que raramente regride (3). Na maioria dos casos, é diagnosticada após a retirada da lesão, através do estudo anatomopatológico do útero. Os aspectos ecográfico, tomográfico e angiográfico desta patologia devem ser diferenciados de outras lesões uterinas, tais como mola parcial, mioma intramural com alterações degenerativas, hemangiossarcoma e hemangiopericitoma.

\section{Relato de caso}

Mulher, 37 anos, casada, nulípara, menarca aos 15 anos, não utilizando nenhum método contraceptivo e possuindo o diagnóstico de carcinoma lobular invasor na mama esquerda. A paciente foi internada no serviço de Oncologia do Hospital Geral de Caxias do Sul para realizar estadiamento da neoplasia mamária, a fim de iniciar tratamento quimioterápico, com ciclos de adriamicina e ciclofosfamida em caráter neo-adjuvante, para posterior ressecção cirúrgica. Durante o estadiamento sistêmico da doença mamária, evidenciou-se, por meio do estudo ultrasonográfico uterino, ecotextura miometrial heterogênea, com a presença de uma massa intramural com neovascularização medindo $10 \mathrm{~cm}$ de diâmetro. $O$ endométrio media $13 \mathrm{~mm}$ de espessura. A paciente tinha história de infertilidade e possuía abundante fluxo menstrual.

Foi realizada uma curetagem semiótica, para definir o tratamento cirúrgico, que resultou em um diagnóstico inconclusivo por apresentar material insuficiente e abundante quantidade de coágulos hemáticos. Durante o procedimento deste método diagnóstico, observou-se profuso sangramento uterino. Mediante este quadro, a paciente foi submetida a histerectomia.
1. Médica patologista do Hospital Ceral de Caxias do Sul-RS; médica patologista do laboratório Diagnose; professora da disciplina de Anatomia Patológica da Universidade de Caxias do Sul. 2. Acadêmica do $11^{\circ}$ semestre do curso de Medicina da Universidade de Caxias do Sul. 
Macroscopicamente, o útero era piriforme pesando $235 \mathrm{~g}$ e medindo $14,7 \mathrm{~cm} \times 7,5 \mathrm{~cm} \times 5,5 \mathrm{~cm}$. A serosa era lisa e brilhante. Aos cortes, o miométrio mostrava-se pardacento, com aspecto esponjoso, medindo $2,4 \mathrm{~cm}$ de espessura. $\mathrm{O}$ endométrio apresentava-se pardo claro e homogêneo, exibindo pólipo ístmico pardo claro e medindo $2,4 \mathrm{~cm}$. O colo uterino media $3,1 \mathrm{~cm}$ de base e $5 \mathrm{~cm}$ de altura. A ectocérvice mostrava-se pardo-clara e lisa. O orifício externo era fendiforme e mostrava junção escamocolunar (JEC) no nível do mesmo. A endocérvice apresentava-se regularmente pregueada, vendo-se formação polipóide ístmica, medindo $2,4 \mathrm{~cm}$ no maior eixo (Figura 1 ).

Microscopicamente, o endométrio mostrava padrão proliferativo, dentro dos limites da normalidade. O miométrio exibia uma ampla rede de vasos, anastomosandose entre si, ocupando dois terços da espessura miometrial em toda a cavidade uterina, sem, no entanto, formar uma massa circunscrita (Figura 2).

\section{Discussão}

O hemangioma cavernoso difuso do útero é uma condição rara na qual a parede uterina é parcialmente ou totalmente substituída por uma grande quantidade de fístulas arteriovenosas de aparência cavernosa, assemelhando-se a uma esponja.

Quando a lesão é mais circunscrita, é referida como hemangioma. A diferença entre hemangioma e hemangioma cavernoso não é clara, sendo possivelmente estágios transicionais. A malformação, nessa doença, resulta da proliferação de vasos arteriais e venosos de tamanhos variados, com formação fistular entre si, os quais vão gradualmente substituindo o miométrio normal. A lesão pode ser congênita ou adquirida. Casos adquiridos estão associa-

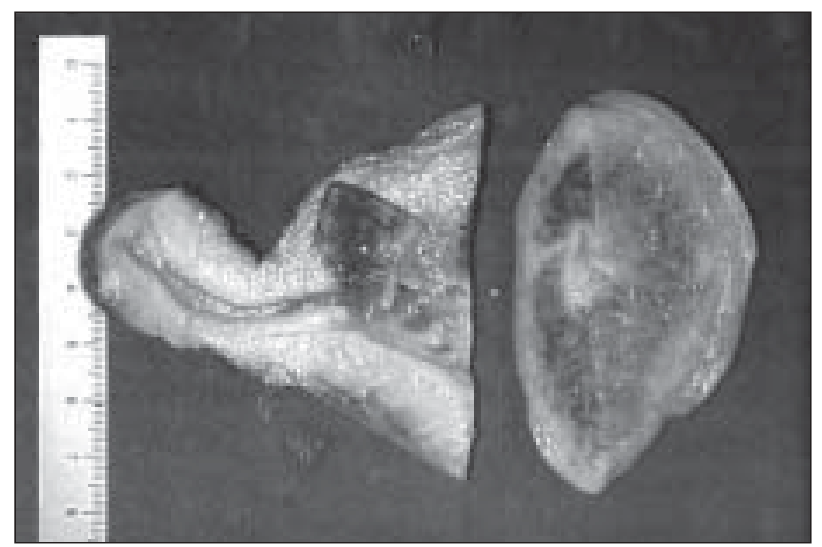

Figura 1 - Peça anatômica mostrando a parede miometrial sendo invadida por vasos sangüíneos dos a cirurgias prévias, curetagens uterinas, doença trofoblástica, carcinoma endometrial e ingestão materna de dietilstilbestrol $(2,4)$.

Em alguns casos, podem existir áreas semelhantes a lesões de um hemangioma capilar em achados focais e, muitas vezes, os hemangiomas cavernoso e capilar podem coexistir numa mesma região $(5,10)$. Trombose, calcificações distróficas secundárias e leve grau de inflamação são achados freqüentes.

A apresentação clínica do hemangioma cavernoso difuso do útero é altamente variável. Alguns relatos $(2,6)$ descrevem a presença de murmúrios, pulsações, sopros ou um útero com aparência esponjosa; enquanto há casos em que há falta de achados clínicos. O mais comum é haver presença de sinais hemorrágicos durante a curetagem uterina, como ocorreu no caso clínico acima descrito, quando o delgado tecido endometrial, que recobre o hemangioma, é removido, sendo expostos os vasos sangüíneos. O fluxo sangüíneo na lesão pode ser avaliado pela ultra-sonografia com doppler colorido. Calcificações em massas uterinas são achados importantes na distinção do hemangioma cavernoso com outras lesões. A tomografia computadorizada (TC) obtida sem contraste descreve uma grande massa pouco atenuada. A ressonância magnética (RM) mostra-se de ajuda no diagnóstico de grandes massas uterinas. Na RM, observa-se uma massa substituindo o miométrio, sendo que o endométrio e o epitélio vesical permanecem inalterados. Em TC, os hemangiomas cavernosos difusos são homogêneos e possuem um sinal de alta intensidade. A aparência angiográfica - densa turvação do material contrastado - com prolongada estase é característica do hemangioma cavernoso. Apesar da acurácia destes métodos diagnósticos, na maioria dos casos o diagnóstico desta patologia é comumente feito com o estudo anatomopatológico do espécime, que revela uma impregnação sangüínea miometrial de aparência esponjosa.

O hemangioma cavernoso difuso do útero deve ser diferenciado de condições como mola parcial, mioma intramural com mudanças degenerativas e de lesões contendo sangue (hemangiossarcoma, hemangiopericitoma) (7). Estas lesões são tipicamente circunscritas e não têm aparência cavernosa.

O maior risco do hemangioma cavernoso difuso do útero é a incerteza ou o erro diagnóstico, podendo complicar o tratamento da paciente (8). Os resultados são laparotomias desnecessárias com subseqüentes hemorragias, que demonstram a importância da acurácia do diagnóstico desta condição. 


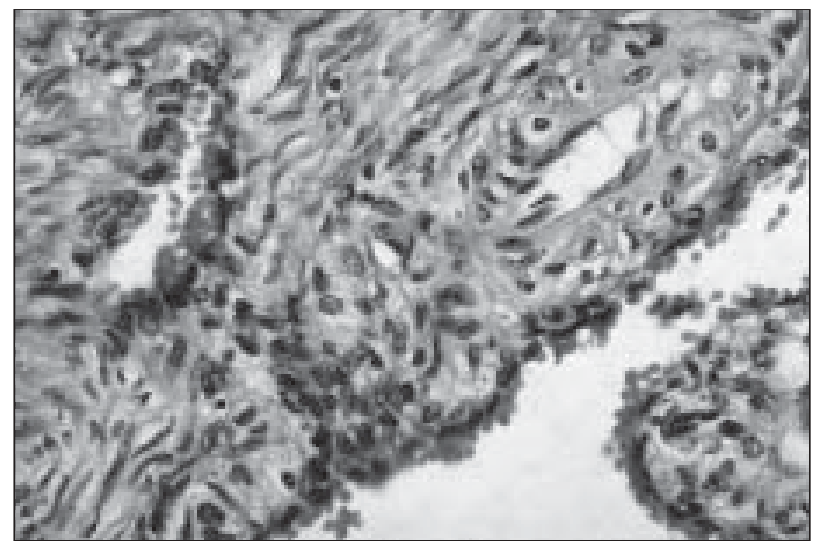

Figura 2 - Aspecto histológico demonstrando hemangioma cavernoso com proliferação endotelial no tecido intramural uterino (400x)
Muitos casos são resolvidos com histerectomia, na tentativa de controle do sangramento profuso. Pode-se utilizar também a embolização das artérias uterinas e a ligação cirúrgica da irrigação vascular com posterior excisão da lesão. Após estes procedimentos, o fluxo sangüíneo decresce e a hemorragia cessa.

Hemangiomas cavernosos difusos do útero foram descritos durante a gravidez, podendo, em alguns casos, ocasionar problemas durante o parto (9).

O prognóstico das pacientes com este tipo de lesão é favorável, havendo uma melhora do quadro após a excisão da lesão.

\section{Referências}

1. McBurney, R.P. \& Trumbull, M. Hemangio ma of the ovary with ascites. M ississippi Doct., 32: 271-4, 1955.

2. Frencken, V.A.M. \& Landman, G.H.M. Ciroid aneurysm of the uterus: Specific arteriographic diagnosis. Am. J. Radiol., 95: 775-81, 1965.

3. Fletcher, C .D .M \& \& C alonge, E. Diagnostic histopathology of tumors. 2 ed. Churchill Livingstone, 2000. p. 52-3.

4. Diwan, R.V. et al. Sonographic diagnosis of arteriovenous malformations of the uterus and pelvis. JUC, 11:295-8, 1983.

5. Kurman, R.J. Blaustein's Pathology of the female genital tract. 4 ed. Springer Verlag, 1994. p. 520-1.
6. Lotgering,F.K. et al. Pregnancy in a patient with diffuse cavernous hemangioma of the uterus. Am.J. Obstet. Gynecol., 160:62830, 1989.

7. Stark, D.D.\& Bradley,W .G. Jr. M agnetic resonance imaging. Vol. 2. St. Louis: Mosby-Year Book, 1992. p. 975-1715.

8.W eissman, A.;Talmon, R. \& Jakobi, P. C avernous hemangioma of the uterus in a pregnant woman. Obstet. Gynecol., 81:8257, 1993.

9. Fleming, $H$. et al. Arteriovenous malformations of the uterus. Obstet. Gynecol., 73: 209-13, 1989.

10.A lvarez, M \& \& C erezo, L. 0 varian cavernous hemangioma. Arch. Pathol. Lab. Med., 110: 77-8, 1986. 